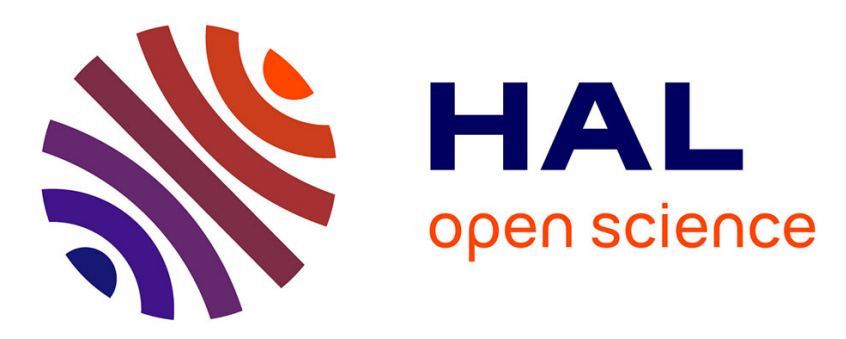

\title{
L'expérience particulière du Japon en matière de renouvellement urbain
}

Natacha Aveline-Dubach

\section{To cite this version:}

Natacha Aveline-Dubach. L'expérience particulière du Japon en matière de renouvellement urbain. Journée d'études sur le renouvellement urbain, Oct 2002, Toulouse, France. pp.59-69. halshs00393968

\section{HAL Id: halshs-00393968 \\ https://shs.hal.science/halshs-00393968}

Submitted on 18 Jun 2009

HAL is a multi-disciplinary open access archive for the deposit and dissemination of scientific research documents, whether they are published or not. The documents may come from teaching and research institutions in France or abroad, or from public or private research centers.
L'archive ouverte pluridisciplinaire HAL, est destinée au dépôt et à la diffusion de documents scientifiques de niveau recherche, publiés ou non, émanant des établissements d'enseignement et de recherche français ou étrangers, des laboratoires publics ou privés. 


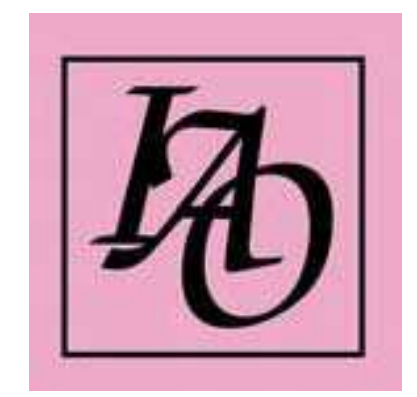

MANUSCRIT DE L'AUTEUR

Référence:

Natacha Aveline, «L'expérience particulière du Japon en matière de renouvellement urbain ", Droit et Ville, ${ }^{\circ} 55,2003$, actes de la journée d'études sur le renouvellement urbain du 18 oct. 2002, p.59-69.

NATACHA AVELINE

L'EXPERIENCE PARTICULIERE DU JAPON EN MATIERE

DE RENOUVELLEMENT URBAIN

IEJUC, TOULOUSE, 2002 
Au Japon, le renouvellement des constructions s'effectue à une cadence sans équivalent dans le monde. La récurrence des désastres naturels dans cette zone à très forte sismicité n'y est incontestablement pas étrangère. Habitués aux ravages des séismes, éruptions volcaniques, typhons ou raz-de-marée, les Japonais en sont venus tout naturellement à considérer leurs constructions comme éphémères. Toutefois, le déterminisme géographique ne saurait être poussé trop avant, car le geste de reconstruire au Japon est loin d'être associé aux désastres. Profondément ancré la tradition religieuse, avec le renouvellement rituel, tous les trente ans, des grands sanctuaires shintoïstes de la région d'Ise, il est un véritable fait de civilisation. Au point que dans les grandes zones urbaines, la durée de vie d'une maison ou d'un immeuble dépasse rarement les cinquante ans.

$\mathrm{Au}$ cours des deux dernières décennies, les autorités ont tenté d'apporter un peu de cohérence au renouvellement anarchique du bâti. Les années 1986-1990 ont connu une politique de «renaissance urbaine » (toshi runesansu), destinée à placer Tôkyô au rang d'une capitale mondiale. Il s'agissait de surmonter l'extrême morcellement de la propriété foncière pour permettre la construction de grands immeubles de bureaux et, dans une moindre mesure, de logements. De grandes opérations de remembrement foncier, impliquant plusieurs centaines d'ayants-droit, ont alors été lancées pour doter la capitale de bâtiments et d'infrastructures aux normes internationales. Cette période a coïncidé avec une hausse spectaculaire des valeurs foncières dans les arrondissements du centre de Tôkyô, induite par le relâchement des conditions d'attribution du crédit bancaire et par la déréglementation des règles d'urbanisme et de construction.

La surchauffe des marchés immobiliers a incité les autorités à se focaliser d'avantage sur les effets désastreux de la spéculation foncière, dont elles étaient, soit dit en passant, largement responsables. La décennie $1990 \mathrm{a}$ donc été marquée par l'élaboration d'une série de mesures destinées à pénaliser la rétention des sols pour augmenter l'offre de terrains résidentiels. C'est également au cours de cette période que les prix fonciers ont commencé à baisser, moins sous l'effet des mesures gouvernementales qu'en raison d'une surproduction de bureaux.

$\mathrm{Au}$ terme d'une décennie d'érosion des valeurs foncières, le système financier dans son ensemble se trouvait fragilisé en raison des énormes volumes de crédit qui avaient été nantis sur des terrains au cours des années d'euphorie financière. C'est dans ce contexte qu'a été formulée, en 2001, une nouvelle politique urbaine baptisée "politique de renouvellement urbain » (toshi saisei). On pourrait penser qu'il s'agit, comme en France, d'un nouveau dispositif destiné à renouveler la ville par des interventions moins lourdes et plus respectueuses de l'existant, mais il n'en est rien. La tabula rasa est toujours de rigueur, c'est le contexte de l'intervention urbaine qui a radicalement changé. Alors que la « renaissance urbaine » des années 1980 visait à faciliter l'accès au foncier pour les promoteurs privés dans un contexte de pénurie et de cherté des terrains, le «renouvellement urbain » s'inscrit, deux décennies plus tard, dans un cadre d'abondance foncière et de chute des prix (jusqu'à 70\% depuis 1991 dans les quartiers d'affaires du centre). 


\section{Les facteurs de l'excédent d'offre foncière}

Pourquoi un excès de terrains à Tôkyô, alors qu'un phénomène de ce genre aurait été impensable une décennie plus tôt ? Trois facteurs se conjuguent pour provoquer cette surabondance foncière.

En premier lieu, il faut bien évidemment mentionner les parcelles qui n'ont pu être construites après le constat, par les promoteurs, de l'ampleur de la surproduction immobilière. Une grande partie de ces terrains étaient détenue par des marchands de biens. Ce sont en effet ces opérateurs, nommés jiage-ya, qui procédaient aux remembrements de petites parcelles pour céder aux promoteurs de grands terrains, empochant de fortes plus-values en raison du différentiel de densité autorisée ${ }^{1}$. Lorsque les promoteurs ont ralenti leur activité, les jiage-ya se sont retrouvés «collés» avec des petites parcelles disséminées dans plusieurs périmètres d'intervention et pour lesquelles ils avaient contracté des emprunts. L'effondrement de la demande, conjuguée à un brusque relèvement des taux d'intérêt, a provoqué l'insolvabilité d'un grand nombre de ces petits opérateurs. On trouve ainsi, dans plusieurs quartiers de Tôkyô, d'anciens périmètres de remembrement en voie de délabrement, où des terrains en friche, souvent convertis en emplacements de parking ${ }^{2}$, voisinent avec des habitations vétustes, parfois squattées par des sans-abri.

Le second facteur d'abondance foncière est la libération de friches industrielles le long du littoral (les zones industrielles de Tôkyô y étant concentrées) et aux abords de grandes voies d'eau comme la rivière Sumida. Ce mouvement s'est accéléré au cours de la dernière décennie, en raison d'un phénomène de rattrapage dans l'industrie des services et de la délocalisation des entreprises japonaises dans d'autres régions d'Asie.

Enfin, on assiste également depuis peu à la libération de plusieurs très grandes friches ferroviaires dans le centre de Tôkyô. Ces terrains étaient pour la plupart détenus par l'ancienne compagnie ferroviaire publique, la JNR (Japan National Railways), privatisée en 1987. L'Etat avait tenté de vendre quelques-unes de ces friches, dès la fin des années 1980, pour combler le déficit de la compagnie publique. Mais la mise aux enchères de ces grandes parcelles très bien placées, dans un contexte de pénurie foncière, avait provoqué une diffusion de la hausse des prix dans les quartiers adjacents. Accusé par la presse d'attiser la flambée foncière, le gouvernement avait donc été contraint de suspendre provisoirement la vente des fiches de la JNR. Celles-ci n'ont pu être mises sur le marché qu'après la baisse des valeurs foncières, c'est-à-dire à la fin des années 1990.

À la différence des friches industrielles, situées dans des zones périphériques à l'environnement peu valorisant, ces friches ferroviaires offrent de belles perspectives pour la promotion immobilière. Elles sont en effet réparties le long de la ceinture ferroviaire Yamanote qui circonscrit le centre-ville et dessert les plus grandes gares du pays. Les gares sont au Japon plus que de simples lieux de transit. Elles constituent les noyaux structurants d'une agglomération où le chemin de fer assure la base des chaînes de déplacements

${ }^{1}$ Du fait de leur exiguité, les petites parcelles ne peuvent faire l'objet de construction d'un grand gabarit même si le plan d'occupation des sols l'autorise. Les jiage-ya achetaient donc des petits terrains à faible prix à des particuliers et revendaient les terrains remembrés au prix fort aux promoteurs.

${ }^{2}$ Une parcelle utilisée, même provisoirement, comme espace de parking est exonérée de taxe foncière si les emplacements sont marqués à la peinture. Cette occupation apporte, en outre, des revenus d'exploitation non négligeables. 
urbains. Elles sont dotées, en outre, de tous les attributs de la centralité, avec leurs grands magasins, hôtels, bureaux et équipements culturels en tout genre. On image bien, par conséquent, les enjeux économiques et urbanistiques associés à l'aménagement des friches de l'ex-JNR.

\section{Les conséquences de l'excédent d'offre foncière}

Venons-en maintenant aux conséquences de l'abondance foncière. Pour ce qui concerne les petites parcelles inscrites dans des périmètres de remembrement, le problème est avant tout financier. Certes, le blocage des opérations a induit une dégradation très sensible du paysage urbain en certains endroits, mais le souci des pouvoirs publics porte avant tout sur le maintien des grands équilibres économiques. Il s'agit donc dans ce cas de débloquer les opérations pour enrayer la dégradation de valeurs foncières et libérer les banques de leurs mauvaises créances.

Le cas des friches industrielles est différent, car ce sont de grandes parcelles n'exigeant pas de lourds processus de remembrement. Quand leur localisation le permet, elles sont le plus souvent converties en opérations de logements, dont la densité n'est d'ailleurs pas sans évoquer celle de nos ZUP dans leurs heures de gloire. Bien que les immeubles soient peu avenants, les appartements se vendent sans difficulté en raison de leur relative proximité au centre et de leurs prix attractifs. Toutefois, nombreuses sont les parcelles impropres à de telles reconversions. Dans ce cas, les terrains demeurent en friche ou accueillent des dépôts illégaux d'ordures, en particulier des gravats de bâtiments démolis.

Il en va tout autrement des friches ferroviaires de l'ex-JNR, appelées à devenir de nouveaux centres urbains ou à renforcer les centres existants, avec l'aménagement de grandes opérations complexes logements/bureaux/commerces. Toute la partie est l'est de la ligne Yamanote est concernée par la conversion de ces friches, soit du Nord au sud : Akihabara (future «Silicon Alley » de 8,8 hectares), la gare de Tôkyô, Shimbashi/Shiodome (ancienne gare de fret ferroviaire) où se construit la plus grande opérations actuelle du pays sur 31 hectares, et Shinagawa où deux grands projets (respectivement de 17 ha et 7,2 ha), en partie sur des friches ferroviaires, accompagneront la construction d'une gare de Shinkansen.

\section{Les objectifs de la politique de renouvellement urbain}

On le voit, les enjeux économiques et urbanistiques sont très différents selon la nature des terrains considérés. Comment, dans ces conditions, a été formulée la nouvelle politique dite de « renouvellement urbain »?

À la lecture des documents officiels, les intentions apparaissent passablement difficiles à cerner. Trois grandes catégories d'objectifs peuvent néanmoins être distinguées. Il s'agit tout d'abord de la requalification des quartiers de maisons et bâtiments en bois, qui ceinturent la partie externe de la ligne Yamanote (carte). Longtemps ignorés des politiques publiques en raison de leur relatif éloignement, occupés par des personnes âgées modestes ou des populations immigrées asiatiques, ces quartiers d'habitats vétustes se retrouvent aujourd'hui à proximité du centre et offrent donc de bonnes perspectives de valorisation foncière. La politique officielle insiste sur les risques auxquels sont exposés ces quartiers en cas de sinistre (matériau bois inflammable, voies trop étroites pour permettre le passage de voitures de pompiers...) et préconise leur reconstruction, associée à l'aménagement d'une nouvelle rocade. Toutefois, aucune politique 
d'accompagnement n'est prévue pour reloger les habitants. Comme partout ailleurs, seuls les ayants droit fonciers (propriétaires ou titulaires d'un bail foncier) seront indemnisés ou sollicités pour participer aux projets de reconstruction ${ }^{3}$.

Le second objectif consiste à promouvoir la mixité des fonctions urbaines. Ne nous y trompons pas : il ne s'agit pas ici de mixité sociale comme en France mais bien de mixité des fonctions. Au cours de la période d'euphorie immobilière des années 1980, la construction de bureaux a été privilégiée dans le centre au détriment des logements. Les résidents des arrondissements centraux ont donc migré vers les périphéries urbaines, avec les conséquences que l'on peut imaginer: fermeture d'écoles, dévitalisation des quartiers, dislocation des liens communautaires, érosion des ressources fiscales pour les mairies d'arrondissement. Aussi, la nouvelle génération de projets cherche-t-elle à décliner diverses fonctions urbaines (bureaux, logements, commerces) tout en valorisant les espaces de la vie sociale (promenades, équipements culturels). Le maitre mot est le rapprochement domicile-travail, à l'inverse des opérations précédentes qui avaient conduit à un allongement conséquent des migrations pendulaires. Les logements en question sont cependant loin d'être accessibles au tout venant. Qui peut en effet se permettre de consacrer 80 millions de yens (soit 619790 euros) pour un appartement trois pièces de 85 mètres carrés?

Le troisième objectif est d'enrayer la dégradation des valeurs foncières, là où les enjeux pour le système financier sont les plus cruciaux. C'est pourquoi la politique de renouvellement porte essentiellement sur le centre et le péri-centre des grandes villes -et tout particulièrement de Tôkyô-, délaissant les secteurs de la grande banlieue, pourtant en proie à un grave phénomène de « déprise».

\section{Les enjeux véritables de la politique de renouvellement urbain}

Il apparaît très clairement, par conséquent, que cette politique de renouvellement urbain cherche d'abord à résorber la grave crise financière qui sévit au Japon depuis plus de dix ans. Elle répond en outre admirablement aux nouveaux besoins de la promotion immobilière privée.

Reprenons en effet les termes de cette politique : s'agissant de la requalification de la ceinture d'habitations en bois, elle peut être considérée comme une avancée en matière de prévention des sinistres et d'amélioration du confort des logements, mais la majeure partie des occupants actuels n'en profiteront pas. Promoteurs et ayants droit se partageront la «rente foncière », tandis que ceux qui ne peuvent prétendre à des indemnités devront quitter le quartier. Ils seront d'ailleurs bien en peine de se reloger ailleurs aux mêmes conditions, puisque le «parc social de fait», indispensable dans une agglomération où l'offre de logements sociaux est très faible, se trouve précisément concentré dans ces quartiers ${ }^{4}$.

\footnotetext{
${ }^{3}$ L'aménagement dans les zones construites se fait le plus souvent par le biais de la procédure de rénovation urbaine (toshi saikaihatsu) qui permet aux propriétaires d'échanger leur terrain contre des surfaces dans les nouveaux bâtiments (équivalent au système de dation en paiement).

${ }^{4}$ Les logements sociaux sont en petit nombre à Tôkyô. En outre, les ressortissants d'autres pays asiatiques, nombreux dans ces quartiers de constructions en bois, sont traditionnellement exclus du logement social.
} 
Pour ce qui concerne la mixité des fonctions, elle arrive à point nommé, au moment où le marché de bureaux s'essouffle alors que le bas niveau des taux d'intérêt soutient une forte demande de logement. En outre, le retour de la population résidentielle au centre a pour effet d'augmenter la demande de commerces de proximité, restaurants, cafés et équipements culturels. Tout cela est bien évidemment au bénéfice des promoteurs, qui tirent ainsi parti de la diversification de la demande.

Enfin, pour ce qui concerne le soutien aux valeurs foncières, les enjeux économiques et financiers en sont très clairs. Nous allons examiner maintenant de plus près comment les autorités comptent atteindre ce troisième objectif.

\section{Comment contrecarrer l'érosion des valeurs foncières?}

La première démarche a consisté à prendre la mesure de la surabondance foncière. Un inventaire des terrains inutilisés ou insuffisamment exploités a été engagé auprès du grand public par le biais du réseau Internet. Tout propriétaire peut accéder à cette «banque foncière » virtuelle pour mettre une parcelle sur le marché. À ce jour, 661 terrains couvrant au total 362 ha ont été enregistrés au niveau national. Un tiers de ces surfaces $(113 \mathrm{ha})$ a fait l'objet de transactions, pour une grande majorité à Tôkyô (92 ha, contre 13 ha à Osaka et 2 à Nagoya).

Le tableau 1 apporte quelques éléments d'information sur les montants échangés. Dans les trois arrondissements centraux de Tôkyô, où les valeurs ont le plus chuté, les prix moyens se sont élevés à 13,490 millions de yens au mètre carré (104 500 euros $/ \mathrm{m} 2)$.

Deux organismes ont été désignés par le gouvernement pour activer le renouvellement urbain. Le plus important est la Urban Development Corporation (toshi seibi kôdan), organisme public opérant à l'échelon national et principal aménageur des villes nouvelles. La UDC a lancé, en 1998, un programme de coopération public-privé dont la vocation est double : débloquer les opérations dans les secteurs de remembrement où des jiage-ya sont intervenus, et requalifier les friches industrielles. Dans le premier cas, la UDC rachète tout ou partie des droits fonciers dans les périmètres considérés, redécoupe le parcellaire en plus grands lots, puis cède ceux-ci à des promoteurs privés ou publics (figure 1). Le remodelage du parcellaire permet la construction d'immeubles de plus grande taille que sur les terrains morcelés, ce qui minimise les pertes à la revente. Dans le cas des friches industrielles, le mécanisme est similaire, mais le réaménagement produit de façon plus systématique de grands immeubles de logements, parfois en plein tissu industriel (figure 2).

Deuxième organisme impliqué, le OPUD (Organization for Promoting Urban Development, Minkan toshi $k i k o ̂$ ) est d'une bien moindre envergure que la UDC. Fondé en 1987, il a surtout pour vocation d'encourager les opérateurs privés à investir dans l'aménagement urbain par l'octroi de prêts bonifiés. Dans le cadre de la politique de renouvellement urbain, il a mis en place un système d'acquisition et de portage foncier, pendant 10 ans, pour le compte d'opérateurs privés. Ceux-ci font construire un bâtiment sur le terrain mis à disposition, qu'ils exploitent pendant dix ans. Au terme de cette période, ils achètent alors le terrain à l'aide d'un prêt bonifié de $0,3 \%$ et bénéficient en outre de considérables exonérations fiscales. Dans ce pays où la charge foncière représente souvent plus de $70 \%$ du coût d'une opération immobilière, ce mécanisme permet de débloquer bien des situations. 
A partir de l'automne 2001, la politique de renouvellement urbain a été affichée parmi les sept priorités nationales pour faire sortir le Japon de la crise. Une Agence de Renouvellement Urbain, placée sous tutelle directe du cabinet du Premier ministre, a été créée en septembre 2001 pour coordonner cette politique.

Quelques mois plus tard, en juin 2002, la loi d'urbanisme a fait l'objet d'une importance révision. La nouvelle loi, dite «loi spéciale de renouvellement urbain» (toshi saisei tokubetsu sochi hô) a introduit des « périmètres spéciaux d'intervention d'urgence » dans les centres des grandes agglomérations japonaises, avec des coefficients d'occupation des sols très élevés. Une autre mesure permet par ailleurs aux aménageurs privés de présenter un projet d'urbanisme dans un périmètre dont ils détiennent la majorité du foncier. Ils bénéficient dans ce cas de procédures d'approbation rapides. Malgré la vive opposition qu'elles ont suscité, ces nouvelles mesures ont été adoptées et mises oeuvre sans difficulté par un gouvernement pressé d'en découdre avec le problème des mauvaises créances. Le système de " périmètres spéciaux » a néanmoins été limité à 10 ans et ne concerne que les zones centrales de Tôkyô, Osaka et Nagoya. À ce jour, 17 périmètres ont été désignés à Tôkyô. Le devenir des occupants y est totalement ignoré (à l'exception des ayants droit fonciers), et il n'est aucunement tenu compte de la préservation du patrimoine existant.

Le gouvernement entend, par cette mesure, stabiliser voire relever les valeurs foncières dans l'hypercentre de Tôkyô. Le raisonnement est simple, voire simpliste: l'augmentation des droits à construire, par des coefficients d'occupation plus élevés, provoque mécaniquement une hausse de valeur du sol. Mais c'est ignorer l'énorme risque encouru, à savoir celui d'une surproduction qui pourrait in fine précipiter les valeurs foncières sous l'effet d'une vacance accrue. Quoi qu'il en soit, cette politique de courte vue aura sans conteste un effet aggravant sur les déséquilibres urbains. Le renforcement de la mixité des fonctions au centre de Tôkyô se fera très vraisemblablement au détriment de la mixité sociale. 